Dept. of Math. University of Oslo

Pure Mathematics

ISBN $82-553-1396-6 \quad$ No. 26

ISSN 0806-2439 July 2003

\title{
THE NORMAL INVERSE GAUSSIAN DISTRIBUTION AND SPOT PRICE MODELLING IN ENERGY MARKETS
}

\author{
FRED ESPEN BENTH AND JŪRATE் ŠALTYTE்-BENTH
}

\begin{abstract}
We model spot prices in energy markets with exponential non-Gaussian Ornstein-Uhlenbeck processes. We generalize the classical geometric Brownian motion and Schwartz' mean-reversion model by introducing Lévy processes as the driving noise rather than Brownian motion. Instead of modelling the spot price dynamics as the solution of a stochastic differential equation with jumps, it is advantageous to model the price process directly from a statistical point of view. Imposing the normal inverse Gaussian distribution as the statistical model for the Lévy increments, we obtain a superior fit compared to the Gaussian model when applied to spot price data from the oil and gas markets. We also discuss the problem of pricing forwards and options and outline how to find the market price of risk in an incomplete market.
\end{abstract}

\section{INTRODUCTION}

When modelling spot price dynamics in energy markets one must account for features like seasonal variation, mean-reversion and large price fluctuations. A popular class of stochastic models is the mean-reversion process introduced by Schwartz [13] for commodities. Since this model assume normally distributed price fluctuations, it seems unsuitable for modelling large changes in spot prices and generalizations are called for.

Most authors start out with the stochastic differential equation defining the Schwartz' dynamics and introduce an additional jump noise term (see e.g. Clewlow and Strickland [8], Eydeland and Wolyniec [10] and Pilipović [11]). The modelling philosophy seems to be that the spot price reverts towards the mean with small price variations modelled by a Brownian motion, while larger price jumps are modelled as a Lévy process. The noise comes in multiplicatively, which makes fitting of the model as a cumbersome task (see Clewlow and Strickland [8] for more on estimation of the jump noise).

We suggest to model (de-seasonalized) spot prices as the exponential of a non-Gaussian Ornstein-Uhlenbeck process. This alternative route to defining a stochastic spot price dynamics is advantageous when fitting the model to data. Our approach is adopted from

Date: July 11, 2003.

Key words and phrases. Energy markets, heavy tails, price jumps, normal inverse Gaussian distribution, Lévy processes, incomplete markets, arbitrage-free pricing of derivatives.

We are grateful to Neil Shephard for kindly providing us with Ox-software for maximum likelihood estimation of generalized hyperbolic distributions. Line Alfarrustad, Ole E. Barndorff-Nielsen and Elisa Nicolato are thanked for fruitful discussions and suggestions. Jūratè Šaltytè-Benth is supported by the Norwegian Research Council under grant NFR: 155120/432. 
Barndorff-Nielsen [2], who models stock prices as the exponential of a Lévy process. In stock markets, the log-returns of assets are frequently heavy tailed violating the normal hypothesis implied by geometric Brownian motion. Barndorff-Nielsen [2] proposes the normal inverse Gaussian distribution as an alternative model for log-returns, which leads to a class of geometric normal inverse Gaussian Lévy processes. The 4 parameters of this distribution can be fitted to data by maximum likelihood estimation, a much simpler procedure than estimating parameters in stochastic differential equation with jumps.

The large price fluctuations frequently observed in energy markets will lead to nonnormal deviations from the long-term mean towards which the prices revert. Following the findings in stock markets, we suggest to model these deviations with the normal inverse Gaussian distribution. Our analysis for oil and natural gas shows that the normal inverse Gaussian distribution is appropriate for modelling the residuals of the logarithmic spot prices. This leads to a dynamics of the spot price being the exponential of an Ornstein-Uhlenbeck process driven by a normal inverse Gaussian Lévy noise, generalizing significantly the results in Benth et al. [5].

Dependencies in the residuals are not accounted for in our model since Lévy processes have independent increments. However, the analysis in this paper can be taken as the first step towards a more complex class of stochastic volatility models described in BarndorffNielsen and Shephard [3] (see also the forthcoming book of the same authors [4]). They model separately the marginal distribution and the autocorrelation structure of the logreturns with the exponential of a drifted Brownian motion, where the volatility is following a non-Gaussian Ornstein-Uhlenbeck process with only positive jumps. The analogue in energy markets could be the exponential of an Ornstein-Uhlenbeck process, where the volatility of the residuals are following a non-Gaussian Ornstein-Uhlenbeck process. The study of these models is outside the scope of the present paper.

We also discuss pricing of forwards and options when the underlying energy spot is modelled with our suggested dynamics. Energy markets are usually considered as incomplete since hedging in the spot is not realizable due to prohibitivly high storage and transaction costs, for instance. Even more, introducing jumps in the models for spots leads to even higher degree of incompleteness. To price derivatives written on energy spots, one needs to take into account the risk preferences of the investors. Traditionally this is described by a market price of risk charged for issuing the derivative. The market price of risk is an additional parameter coming from the equivalent martingale measures. We shall derive these measures using the Esscher transform, which will provide us with time-dependent market prices of risk.

Based on our spot dynamics we can derive explicit forward prices as a function of the characteristics of the Lévy process defining the dynamics. Moreover, given the Lévy process we discuss how one can find the market price of risk by fitting theoretical prices to today's observed forward curve. In many cases this can be done perfectly, but in most natural situations one would search for an approximation of the observed forward curve. Options on spots can not be calculated explicitly. However, we know the characteristic function of the logarithmic spot prices given the Lévy process and the market price of risk. This is the necessary input for a numerical approach for pricing based on the fast Fourier transform. 
The paper is organized as follows: We start with defining the spot price dynamics in Section 2, following up with a data analysis of oil and gas spot prices in Section 3 . In Section 4 we derive the price of forward contracts and discuss the problem of option pricing. The market price of risk is calculated via an Esscher transform, and we provide a recipe for fitting it to today's forward curve. We conclude our analysis in Section 5.

\section{A stochastic Spot PRICE MOdel With LÉvy NOISE}

Let $\left(\Omega, \mathcal{F},\left\{\mathcal{F}_{t}\right\}_{t \in[0, T]}, P\right)$ be a complete filtered probability space which satisfies the usual hypotheses and $T<\infty$. Introduce a Lévy process $L_{t}$ with Lévy-Kintchine representation

$$
L_{t}=\nu t+\sigma B_{t}+\int_{|z|<1} z \widetilde{N}((0, t], d z)+\int_{|z| \geq 1} z N((0, t], d z)
$$

where $B_{t}$ is a standard Brownian motion, $\nu$ a constant, $\sigma>0$ a constant, and $N$ a homogeneous Poisson random measure with compensator $d t \ell(d z)$. The $\sigma$-finite measure $\ell(d z)$ on the Borel sets of $\mathbb{R}$ is called the Lévy measure, and satisfies the conditions $\ell(\{0\})=$ 0 and $\int_{\mathbb{R}} \min \left(1, z^{2}\right) \ell(d z)<\infty$. Alternatively, the Lévy-Kintchine formula can be written as $\mathbb{E}\left[\exp \left(\mathrm{i} \lambda L_{t}\right)\right]=\exp (t \psi(\lambda))$, with $\mathrm{i}=\sqrt{-1}$ and $\psi(\lambda)$ being the cumulant function

$$
\psi(\lambda)=\mathrm{i} \lambda \nu-\frac{\lambda^{2}}{2} \sigma^{2}+\int_{\mathbb{R}} \mathrm{e}^{\mathrm{i} \lambda z}-1-\mathrm{i} \lambda z 1_{|z|<1} \ell(d z) .
$$

Assume $S_{t}$ is the spot price at time $t$, which we model as the stochastic process

$$
S_{t}=\Lambda(t) \mathrm{e}^{X_{t}},
$$

where $\Lambda(t)$ is the seasonality (a non-random function of time). The non-Gaussian OrnsteinUhlenbeck process $X_{t}$ has dynamics

$$
d X_{t}=a\left(m-X_{t}\right) d t+d L_{t},
$$

and initial state $X_{0}=x$. The speed of mean-reversion is given by $a \geq 0$, while $m$ is a positive constant indicating a long-term mean of the process. We easily see from the Itô Formula for semimartingales that

$$
X_{t}=x \mathrm{e}^{-a t}+\frac{m}{a}\left(1-\mathrm{e}^{-a t}\right)+\int_{0}^{t} \mathrm{e}^{-a(t-s)} d L_{s} .
$$

In particular, when $L_{t}=\nu t+\sigma B_{t}$ the process $S_{t}$ reduces to the classical mean-reversion model of Schwartz [13]. Choosing $a=0$ in (2.3), we obtain $X_{t}=L_{t}$ and the spot dynamics becomes the exponential of a Lévy process, generalizing the classical geometric Brownian motion model. Hence, our geometric spot price model (2.2) generalizes geometric Brownian motion and Schwartz' mean-reversion dynamics. We remark in passing that stating the dynamics of the spot price directly in exponential form rather than as the solution of a stochastic differential equation is advantageous when fitting to data.

We shall pay particular attention to the normal inverse Gaussian Lévy process (from here on called the NIG Lévy process). This process was first introduced in a financial 
context by Barndorff-Nielsen [2], and is a flexible and successful model for financial logreturns (see the forthcoming book by Barndorff-Nielsen and Shephard [4]). A process $L_{t}$ is called NIG Lévy process if $L_{1}$ is distributed according to the normal inverse Gaussian (NIG) distribution. The NIG distribution is a 4 parameter family of distributions belonging to the class of generalized hyperbolic distributions. The density function of the NIG distribution is explicitly given as

$$
f(x ; \mu, \alpha, \beta, \delta)=\frac{\alpha \delta}{\pi} \exp \left(\delta \sqrt{\alpha^{2}-\beta^{2}}+\beta(x-\mu)\right) \frac{K_{1}\left(\alpha \sqrt{\delta^{2}+(x-\mu)^{2}}\right)}{\sqrt{\delta^{2}+(x-\mu)^{2}}} .
$$

Here, $\mu \in \mathbb{R}$ is the location of the density, $\beta \in \mathbb{R}$ is the skewness parameter, $\alpha \geq|\beta|$ measures the heaviness of the tails and finally, $\delta>0$ is the scale parameter. The function $K_{1}$ is the modified Bessel function of the second kind and order 1 (see e.g. Abramowitz and Stegun [1]). The NIG distribution arises as a mean-variance mixture model. If $Y \mid \sigma^{2} \sim \mathcal{N}\left(\mu+\beta \sigma^{2}, \sigma^{2}\right)$, and $\sigma^{2} \sim \mathcal{I} \mathcal{G}\left(\delta, \sqrt{\alpha^{2}-\beta^{2}}\right)$, with $\mathcal{I} \mathcal{G}$ denoting the inverse Gaussian distribution, then $Y$ is NIG distributed with parameters $(\mu, \alpha, \beta, \delta)$. Since the density is known explicitly, one can do maximum likelihood estimation of the parameters to fit a NIG random variable to data. The Bessel function $K_{1}$ is rather cumbersome to calculate numerically, and sophisticated optimization methods must be implemented in order to solve the estimation problem in practice. However, using the Ox-language (see [9]) this task becomes straightforward. The density of NIG is a predefined function in $0 \mathrm{x}$, and moreover, the language provides a powerful routine for optimization.

If $L_{1}$ is NIG distributed with parameters $(\mu, \alpha, \beta, \delta)$, then the Lévy measure of $L_{t}$ is given by

$$
\ell(d z)=\frac{\alpha \delta}{\pi|z|} \mathrm{e}^{\beta z} K_{1}(\alpha|z|) d z
$$

Furthermore, the cumulant function is given as

$$
\psi(\lambda)=\mathrm{i} \lambda \mu+\delta\left(\sqrt{\alpha^{2}-\beta^{2}}-\sqrt{\alpha^{2}-(\beta+\mathrm{i} \lambda)^{2}}\right) .
$$

The explicit knowledge of $\psi$ is useful in connection with option pricing.

\section{Data Analysis ANd FitTing of SPOT PRICE MOdels}

We analyse two series of daily spot prices from the energy markets. The first series consists of 1898 daily (working days only) spot prices of Brent oil ranging from 24 September 1992 until 17 February 2000. The time series is plotted in Fig. 1. The second series depicted in Fig. 2 shows a collection of 731 daily $\mathrm{SAP}^{1}$ prices ranging from 1 January 2000 until 31 December 2001.

To find the (non-stochastic) annual seasonality of the energy spot prices, we used a first order Fourier expansion,

$$
\Lambda(t)=c_{1}+c_{2} \cos \left(\frac{2 \pi}{n}\left(t-c_{3}\right)\right)
$$

\footnotetext{
${ }^{1} \mathrm{SAP}$ is short-hand for the System Average Price
} 


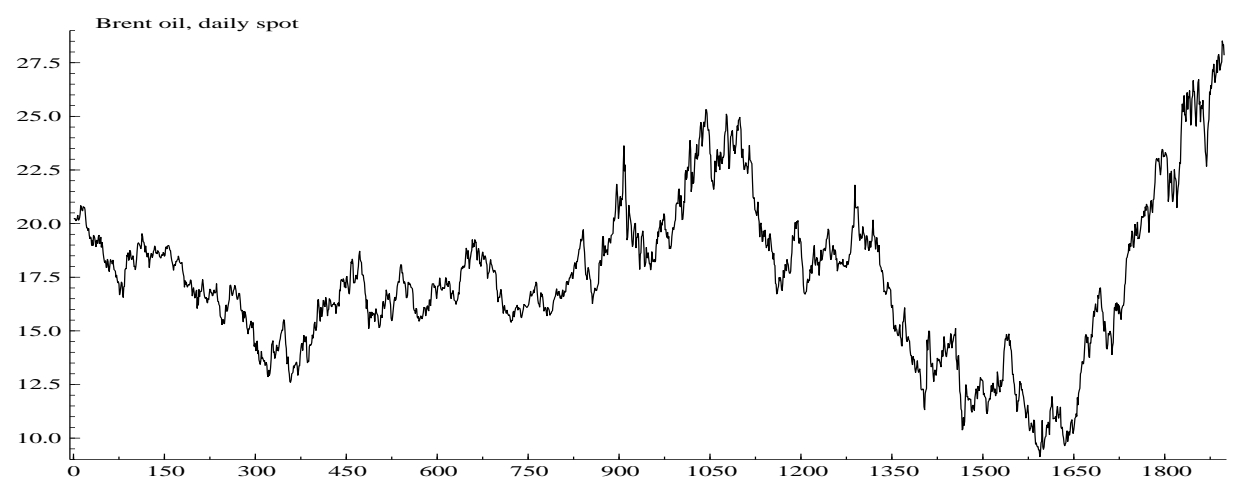

FIGURE 1. Time series plot of the daily Brent oil spot prices

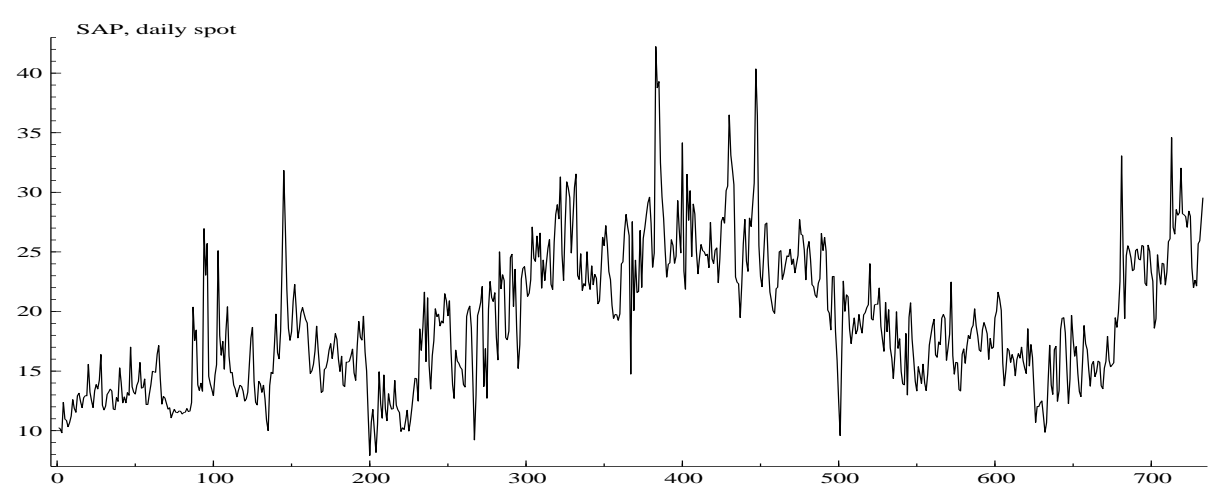

Figure 2. Time series plot of the daily SAP prices

The level $c_{1}$, amplitude $c_{2}$ and time shift $c_{3}$ were all estimated by the method of least squares $^{2}$. The frequency of price quotes was $n=365$ for SAP, while we used an average number of trading days $n=252$ for Brent oil. The analysis gave the parameters $c_{1}=19.14$, $c_{2}=3.23$ and $c_{3}=10.57$ for the SAP data. The oil data, on the other hand, had no significant seasonal pattern, and for this data set we use $\Lambda(t)=1$ from now on.

3.1. Geometric Brownian motion. Our data analysis started out with an investigation of the normal hypothesis for the log-returns from the two series, where we use Brent oil and de-seasonalized SAP spot prices. The estimates for the means and standard deviations $(\mathrm{sdv})$ of the log-returns are exhibited in Table 1. We have calculated the KolmogorovSmirnov statistics and the corresponding significance level, which allowed us to reject the normal hypothesis for the log-returns for both energies at the level $1 \%$. The parameters of the NIG distributions were fitted to the log-return data using maximum likelihood estimation, and the results are presented in Table 2.

\footnotetext{
${ }^{2}$ We used the function nlinfit in Matlab for this purpose.
} 
TABLE 1. Estimated parameters of the normal distribution for the logreturns of the energies

\begin{tabular}{lrr}
\hline & mean & std \\
\hline Brent oil & 0.00017 & 0.021 \\
SAP & 0.00145 & 0.138 \\
\hline
\end{tabular}

TABLE 2. Estimated parameters of the NIG distribution for the log-returns of the energies

\begin{tabular}{lrrrr}
\hline & $\widehat{\mu}$ & $\widehat{\alpha}$ & $\widehat{\beta}$ & $\widehat{\delta}$ \\
\hline Brent oil & 0.00102 & 48.8 & -1.96 & 0.021 \\
SAP & -0.00690 & 5.80 & 0.43 & 0.112 \\
\hline
\end{tabular}

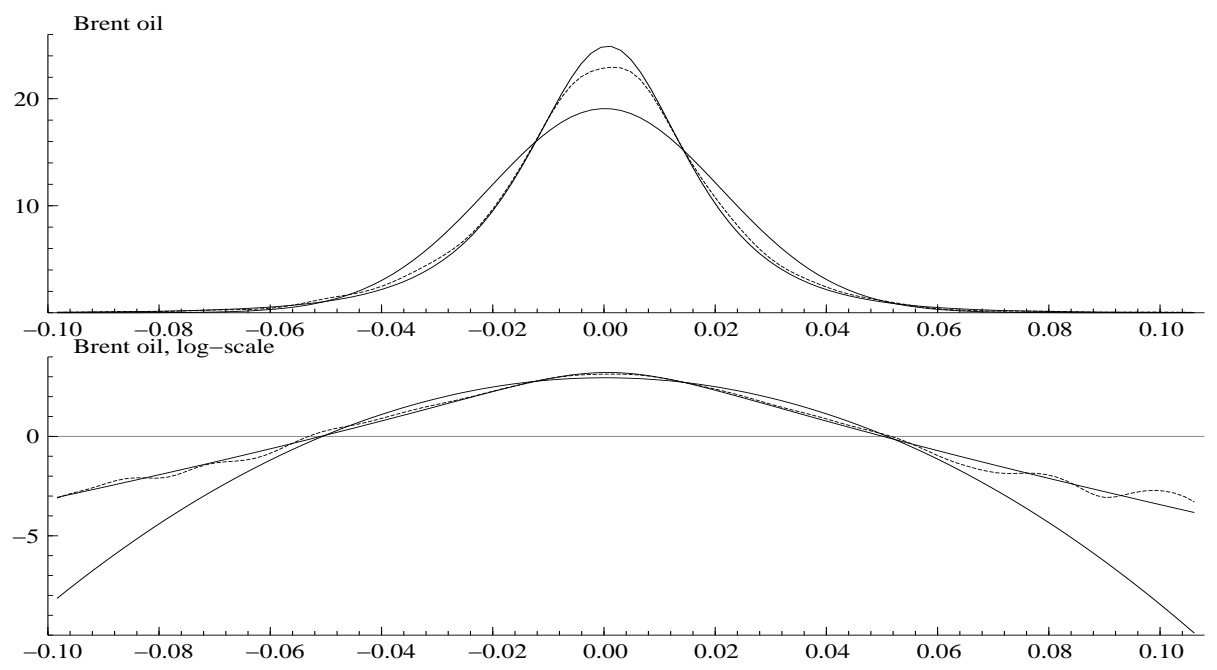

Figure 3. Empirical and fitted densities for Brent oil spot log-returns. The empirical density is plotted using a Gaussian kernel smoother and is the broken line.

Figures 3 and 4 show plots of the empirical densities together with the fitted normal and NIG distributions. We display the densities on a logarithmic scale too, in order to emphasize the tail behaviour. It is evident that the normal distributions are not very good models for the log-returns. The tails are falling off like parabolas, while the empirical densities show almost linear tails on a logarithmic scale. The NIG distributions are catching the observed heavy tails reasonably well. We also see that the normal distributions assign far too little probability in the center compared with the empirical densities. This is corrected in the fitted NIG distributions, although they seem to overestimate a bit. Interestingly, 


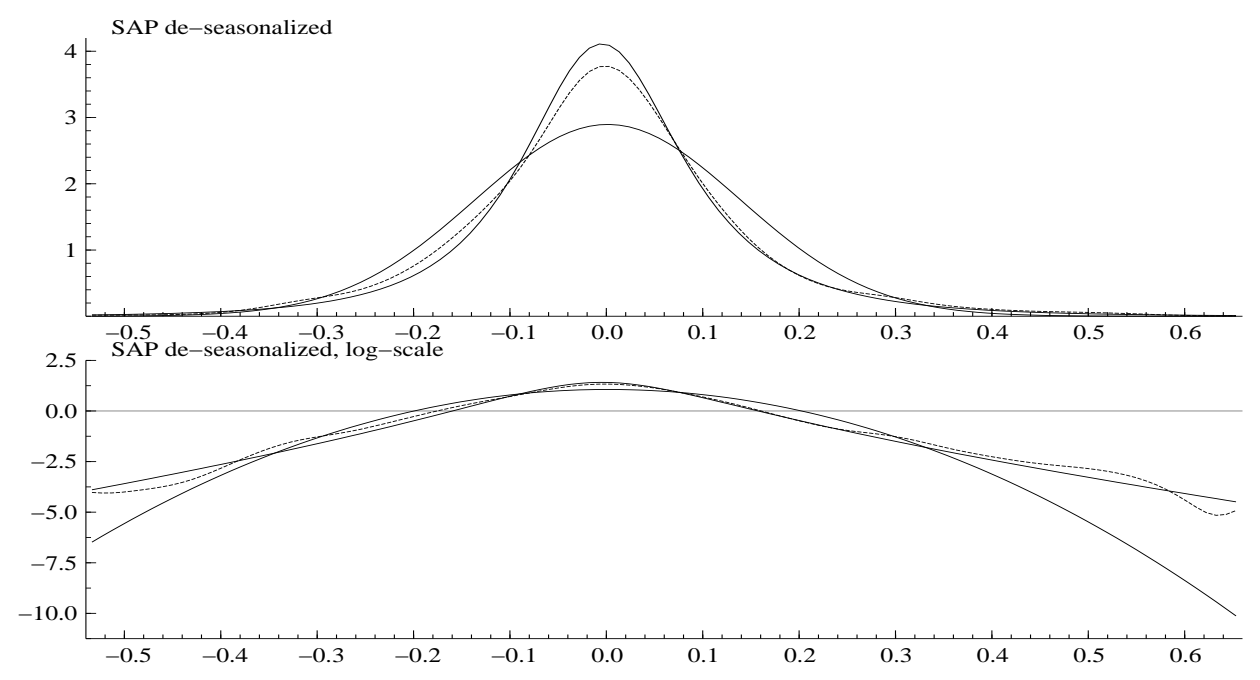

Figure 4. Empirical and fitted densities for the de-seasonalized SAP gas spot log-returns. The empirical density is plotted using a Gaussian kernel smoother and is the broken line.

TABLE 3. Mean reversion parameters

\begin{tabular}{lrrr}
\hline & $\widehat{m}$ & $\widehat{a}$ & $R^{2}$ \\
\hline Brent oil & 2.75 & 0.004 & 0.99 \\
SAP & -0.030 & 0.108 & 0.77 \\
\hline
\end{tabular}

the empirical findings for stock log-returns are similar to what we observe in our analysis (see e.g. [2] and [4]).

To see whether we could improve the fit in the center, we estimated generalized hyperbolic distributions. However, the fitting of the center of the distributions did not become significantly better comparing to the NIG.

3.2. Mean-reversion model. Let us turn our attention to the mean-reversion model with $a>0$. The estimation will go in two steps. First we use linear regression in order to estimate the long-term mean and speed of mean-reversion. Then we investigate the distributional properties of the residuals by fitting the normal and the NIG distributions. Admittedly, one should derive a maximum likelihood estimator for the regression of the latter model. However, it is out of the scope of the present paper to develop such software.

In Table 3 we give the estimated parameters of the mean-reversion models. The estimated speed of mean-reversion for Brent oil is significant at 1\% level, while the long-term mean is only significant at $10 \%$ level. For SAP both estimated parameters are statistically significant at $1 \%$ level. We note that gas has a relatively strong mean-reversion (big value of $\widehat{a}$ ), while $\widehat{m}$ is close to zero ( since we work with de-seasionalized data, this is not surprising). From the values of $R^{2}$ we conclude that the regression line fits data well. 
TABLE 4. Estimated parameters of the normal distribution for the residuals

\begin{tabular}{lrr}
\hline & mean & std \\
\hline Brent oil & -0.000008 & 0.021 \\
SAP & -0.000000 & 0.133 \\
\hline
\end{tabular}

TABLE 5. Estimated parameters of the NIG distribution for the residuals

\begin{tabular}{lrrrr}
\hline & $\widehat{\mu}$ & $\widehat{\alpha}$ & $\widehat{\beta}$ & $\widehat{\delta}$ \\
\hline Brent oil & 0.00091 & 47.1 & -2.11 & 0.020 \\
SAP & -0.01284 & 7.91 & 0.73 & 0.139 \\
\hline
\end{tabular}

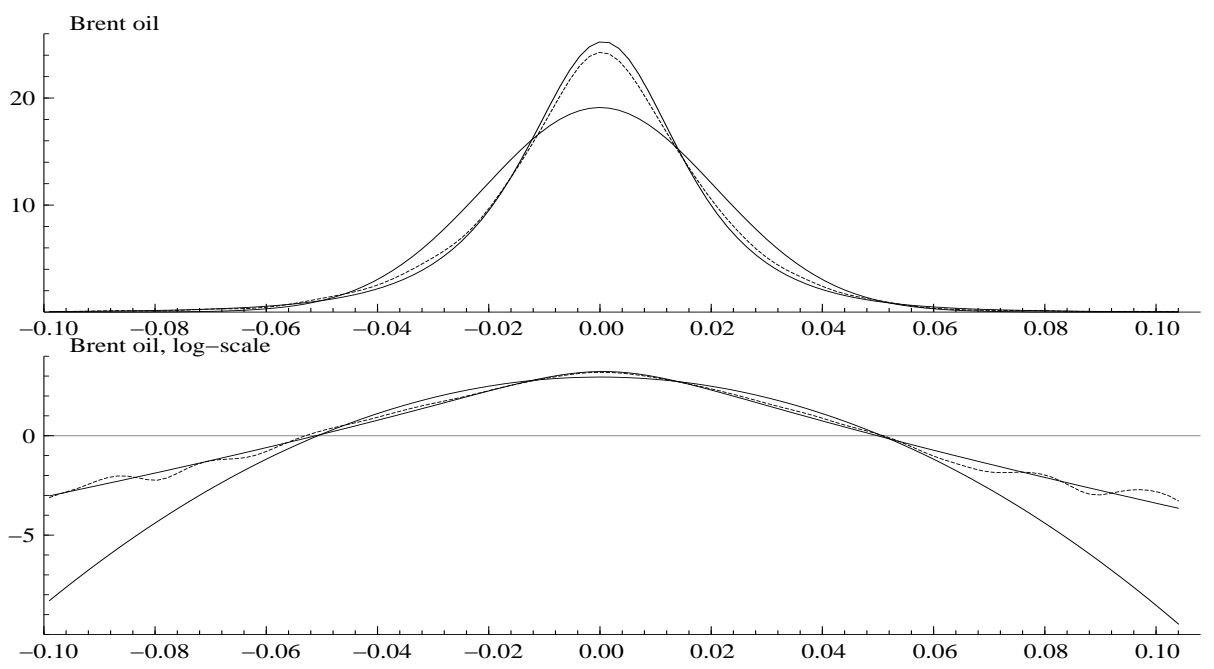

Figure 5. Empirical and fitted densities for Brent oil spot residuals. The empirical density is plotted using a Gaussian kernel smoother and is the broken line.

For the residuals we run the same analysis as for the log-returns and the obtained results are exhibited in Table 4 and Table 5. The normal hypothesis is also rejected at the level $1 \%$.

We have plotted the empirical densities of the residuals together with the fitted normal and NIG distributions for Brent oil and SAP in Figs. 5 and 6 , resp. We reach the same conclusions as for the log-returns of the energies.

\section{Arbitrage-Free pricing of Derivatives}

We include some considerations on pricing of derivatives based on the spot price model $(2.2)$. 


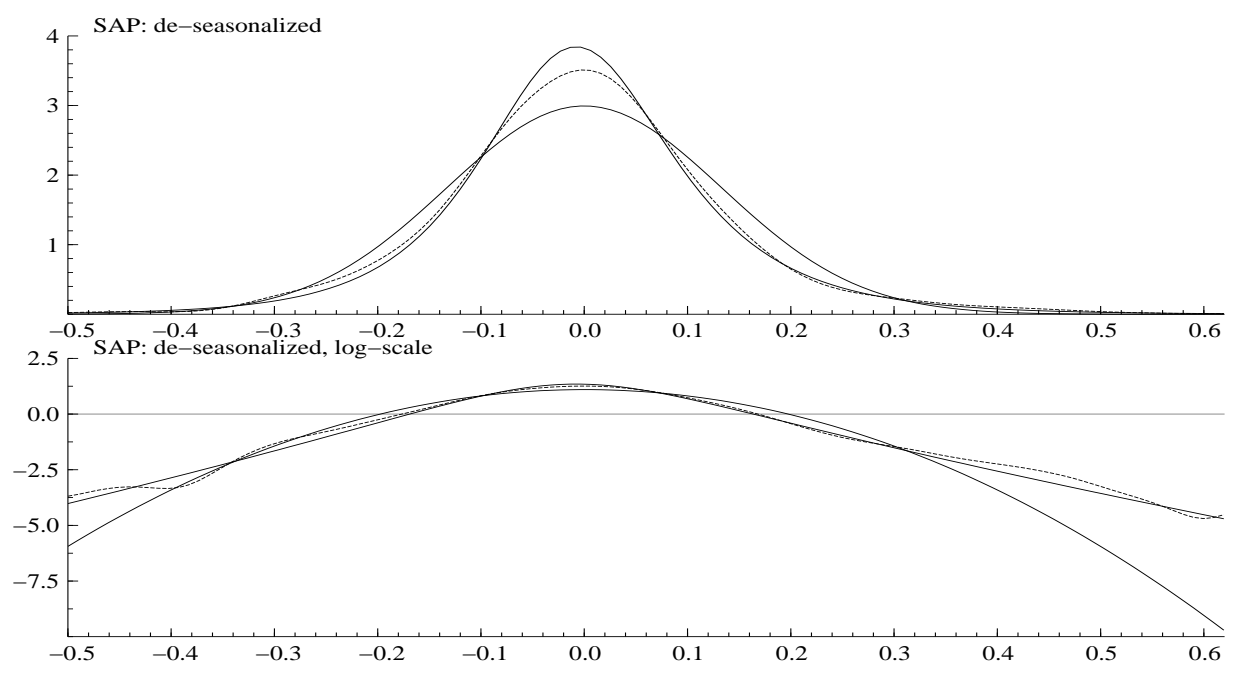

Figure 6. Empirical and fitted densities for the de-seasonalized SAP gas spot residuals. The empirical density is plotted using a Gaussian kernel smoother and is the broken line.

Commodity markets are typical examples of so-called incomplete markets. Trading in a commodity, natural gas say, imposes big transaction and storage costs. Some commodities can not even be stored, like electricity (except possibly indirectly in hydro reservoirs, for instance). These restrictions break down the standard hedging approach used to derive the unique arbitrage-free price for a derivative. As in incomplete financial markets, a continuum of arbitrage-free prices exists, one for each equivalent martingale measure. By definition, an equivalent martingale measure $Q$ is a probability measure equivalent to $P$ and such that all continuously tradeable assets are martingales after discounting. Since typically only the bank account can be traded continuously in the market for a commodity, all equivalent probability measures $Q$ will also be martingale measures. We also consider spot price models with Lévy processes as the driving noise instead of Brownian motion. This adds up the degree of incompleteness of the market.

We shall in this paper derive equivalent measures through the Esscher transform. The usual technique in commodity (and interest-rate markets) to single out one measure $Q$, is to parameterize the class of $Q$ 's and fit the theoretically derived forward curves to the empirical ones.

First, let us impose an exponential integrability condition on the Lévy measure ensuring existence of moments of the spot process. The valuation of forwards and option contracts usually requires the existence of at least the first moment of the underlying.

Condition $(\mathbf{L})$ : There exists a constant $k>0$ such that the Lévy measure satisfies the integrability condition

$$
\int_{1}^{\infty} \mathrm{e}^{k z} \ell(d z)<\infty .
$$


The constant $k$ determines the order of the moments that is finite for the spot price process (2.2). This follows from the next Lemma:

Lemma 4.1. If $g:[0, t] \mapsto \mathbb{R}$ is a bounded and measurable function and Condition (L) holds for $k:=\sup _{s \in[0, t]}|g(s)|$, then

$$
\mathbb{E}\left[\exp \left(\int_{0}^{t} g(s) d L_{s}\right)\right]=\exp \left(\int_{0}^{t} \phi(g(s)) d s\right),
$$

where $\phi(\lambda)=\psi(-i \lambda)$.

Proof. First, let us prove that $\int_{\mathbb{R}}\left|\mathrm{e}^{\lambda z}-1-\lambda z 1_{|z|<1}\right| \ell(d z)<\infty$ for all $|\lambda| \leq k$ : It is sufficient to consider only $\lambda>0$. It follows from the definition of the Lévy measure and Condition (L) that

$$
\begin{aligned}
\int_{\mathbb{R}}\left|\mathrm{e}^{\lambda z}-1-\lambda z 1_{|z|<1}\right| \ell(d z)= & \int_{1}^{\infty}\left|\mathrm{e}^{\lambda z}-1\right| \ell(d z)+\int_{-1}^{1}\left|\mathrm{e}^{\lambda z}-1-\lambda z\right| \ell(d z) \\
& +\int_{-\infty}^{-1}\left|\mathrm{e}^{\lambda z}-1\right| \ell(d z) \\
\leq & \int_{1}^{\infty} \mathrm{e}^{k z} \ell(d z)+K \lambda^{2} \int_{|z|<1} z^{2} \ell(d z)+\int_{-\infty}^{-1} 1 \ell(d z) \\
\leq & C,
\end{aligned}
$$

where $C$ is a constant only dependent on $k$ and not on $\lambda$. From this it follows that since $|g(s)| \leq k$ for all $s \in[0, t]$ the integral $\int_{0}^{t} \phi(g(s)) d s$ is well-defined.

The Lemma now follows from the independent increment property and the Lévy-Kintchine representation of the Lévy process.

Assume $\theta:[0, T] \mapsto \mathbb{R}$ is a measurable and bounded function. Consider the stochastic process

$$
Z_{t}^{\theta}=\exp \left(\int_{0}^{t} \theta(s) d L_{s}-\int_{0}^{t} \phi(\theta(s)) d s\right)
$$

where $\phi(\lambda)$ is the moment generating function of $L_{t}$, e.g. $\phi(\lambda)=\psi(-i \lambda)$. The process $Z_{t}^{\theta}$ is well-defined for $t \in[0, T]$ if Condition (L) holds for $k:=\sup _{t \in[0, T]}|\theta(t)|$. Introduce the probability measure $Q^{\theta}$ defined by the Esscher transform:

$$
Q^{\theta}(A)=\mathbb{E}\left[1_{A} Z_{T}^{\theta}\right]
$$

where $1_{A}$ is the indicator function. This measure is obviously equivalent to $P$, and thus an equivalent martingale measure. Denote the expectation using the probability $Q^{\theta}$ by $\mathbb{E}_{\theta}[\cdot]$. Note that by using time-varying $\theta$ 's we have a more flexible class of martingale measures when fitting to the observed forward curve. The market price of risk is parameterized by $\theta$. We remark in passing that in Benth et al. [5] the market price of risk was derived by a Girsanov shift on the Brownian motion part of a Lévy process. If we model by, for instance, the pure-jump Lévy process defined by the normal inverse Gaussian distribution, 
the market price of risk will be undefined in the setting of [5]. The approach here is much more general and natural.

Let $f$ be a real-valued measurable function with at most linear growth. Then the arbitrage theory tells us that the fair price $P_{t}$ at time $t$ of an option on the spot paying $f\left(S_{T}\right)$ at maturity $T$ is (see e.g. Bjørk [6])

$$
P_{t}=\mathrm{e}^{-r(T-t)} \mathbb{E}_{Q}\left[f\left(S_{T}\right) \mid \mathcal{F}_{t}\right],
$$

for an equivalent martingale measure $Q$. We have used the notation $\mathbb{E}_{Q}[\cdot]$ for the expectation under $Q$. Choosing the equivalent martingale measure $Q^{\theta}$, the following Lemma provides a sufficient condition for the existence of a price dynamics $P_{t}$ :

Lemma 4.2. Suppose Condition (L) holds for $k:=1+\sup _{s \in[0, T]}|\theta(s)|$, then $\mathbb{E}_{\theta}\left[\left|f\left(S_{T}\right)\right|\right]<$ $\infty$.

Proof. First of all, we see that $Z_{T}^{\theta}$ is well-defined from the assumption in the Lemma. From the equivalence of $Q^{\theta}$ and $P$, we find

$$
\mathbb{E}_{\theta}\left[\left|f\left(S_{T}\right)\right|\right]=\mathbb{E}\left[\left|f\left(S_{T}\right)\right| Z_{T}^{\theta}\right]
$$

and by linear growth of $f$ it follows that $\mathbb{E}\left[\exp \left(\int_{0}^{T} \theta(s)+\mathrm{e}^{-a(T-s)} d L_{s}\right)\right]$ must be finite for the Lemma to hold. This is ensured by the assumption.

From now on we suppose that Condition $(\mathbf{L})$ holds for $k:=1+\sup _{s \in[0, T]}|\theta(s)|$ for all $\theta$ 's in question.

4.1. Forward contracts. The arbitrage-free price of a forward contract at time $t$ with delivery at time $T>t$ is defined to be the adapted process $F^{\theta}(t, T)$ such that

$$
0=\mathrm{e}^{-r(T-t)} \mathbb{E}_{\theta}\left[S_{T}-F^{\theta}(t, T) \mid \mathcal{F}_{t}\right] .
$$

Thus,

$$
F^{\theta}(t, T)=\mathbb{E}_{\theta}\left[S_{T} \mid \mathcal{F}_{t}\right]
$$

We derive an explicit formula for the forward price:

Theorem 4.3. The price at time $t$ of a forward contract with delivery at time $T>t$ will be

$$
F^{\theta}(t, T)=\Lambda(T) \exp \left(\int_{t}^{T}\left\{\phi\left(\theta(s)+e^{-a(T-s)}\right)-\phi(\theta(s))\right\} d s\right)\left(\frac{S_{t}}{\Lambda(t)}\right)^{\exp (-a(T-t))}
$$

Proof. First, observe that $X_{t} \mathrm{e}^{-a(T-t)}=X_{T}-\int_{t}^{T} \mathrm{e}^{-a(T-s)} d L_{s}$. Hence,

$$
\begin{aligned}
F^{\theta}(t, T) & =\mathbb{E}_{\theta}\left[S_{T} \mid \mathcal{F}_{t}\right]=\Lambda(T) \mathbb{E}_{\theta}\left[\exp \left(X_{t} \mathrm{e}^{-a(T-t)}+\int_{t}^{T} \mathrm{e}^{-a(T-s)} d L_{s}\right) \mid \mathcal{F}_{t}\right] \\
& =\Lambda(T)\left(\frac{S_{t}}{\Lambda(t)}\right)^{\exp (-a(T-t))} \mathbb{E}_{\theta}\left[\exp \left(\int_{t}^{T} \mathrm{e}^{-a(T-s)} d L_{s}\right) \mid \mathcal{F}_{t}\right] .
\end{aligned}
$$


We continue to calculate the conditional expectation: from Bayes' Formula we get

$$
\begin{aligned}
\mathbb{E}_{\theta}\left[\operatorname { e x p } \left(\int_{t}^{T} \mathrm{e}^{-a(T-s)}\right.\right. & \left.\left.d L_{s}\right) \mid \mathcal{F}_{t}\right] \\
& =\mathbb{E}\left[\exp \left(\int_{t}^{T} \mathrm{e}^{-a(T-s)} d L_{s}\right) \frac{Z_{T}^{\theta}}{Z_{t}^{\theta}} \mid \mathcal{F}_{t}\right] \\
& =\exp \left(-\int_{t}^{T} \phi(\theta(s)) d s\right) \mathbb{E}\left[\exp \left(\int_{t}^{T} \theta(s)+\mathrm{e}^{-a(T-s)} d L_{s}\right) \mid \mathcal{F}_{t}\right] \\
& =\exp \left(-\int_{t}^{T} \phi(\theta(s)) d s\right) \mathbb{E}\left[\exp \left(\int_{t}^{T} \theta(s)+\mathrm{e}^{-a(T-s)} d L_{s}\right)\right] \\
& =\exp \left(\int_{t}^{T}\left\{\phi\left(\theta(s)+\mathrm{e}^{-a\left(T_{s}\right)}\right)-\phi(\theta(s))\right\} d s\right) .
\end{aligned}
$$

This proves the theorem.

We now briefly outline how one can use the today's observed forward curve to find the price of risk $\widehat{\theta}$ chosen by the market. Let $F\left(T_{i}\right), i=1, \ldots, N$, be $N$ prices of forward contracts with delivery time $T_{i}$ resp., where $0<T_{1}<T_{2}<\cdots<T_{N}$. The theoretical forward prices are given by letting $t=0 \mathrm{in}$ Thm. 4.3. Hence, for the forward contract with delivery at time $T_{i}$ we have

$$
F^{\theta}\left(0, T_{i}\right)=\Lambda\left(T_{i}\right) \exp \left(\int_{0}^{T_{i}} \phi\left(\theta(s)+\mathrm{e}^{-a\left(T_{i}-s\right)}\right)-\phi(\theta(s)) d s\right)\left(\frac{S_{0}}{\Lambda(0)}\right)^{\exp \left(-a T_{i}\right)},
$$

$i=1, \ldots, N$. We can now find the $\widehat{\theta}$ which minimizes the distance between the theoretical and observed prices in some appropriate norm, that is,

$$
\widehat{\theta}(\cdot):=\arg \min _{\theta(\cdot)}\left\|F^{\theta}(0, T .)-F(T .)\right\|,
$$

where we minimize over measurable functions $\theta$ being uniformly bounded by a constant. For some specifications we can obtain an exact match between theoretical and observed prices (see e.g. [5]).

4.2. Options. The arbitrage-free price of an option with payoff $f\left(S_{T}\right)$ at maturity $T$ is given in (4.2). To find the price at $t=0$, we need to calculate

$$
P_{0}=\mathrm{e}^{-r T} \mathbb{E}_{\theta}\left[f\left(\Lambda(T) \mathrm{e}^{X_{T}}\right)\right] .
$$

Assume that the market price of risk is specified (for instance by fitting it to the observed forward curve as described in the Subsection above). In order to find the price of the option we need to calculate the expectation. The problem we are facing however, is that we do not know explicitly the distribution of $X_{T}$ under the probability $Q_{\theta}$, except for some very special cases. What we know, however, is its characteristic function: 
Corollary 4.4. For $\lambda \in \mathbb{R}$ it holds $\mathbb{E}_{\theta}\left[\exp \left(i \lambda X_{T}\right)\right]=\exp (\Psi(\lambda))$, where

$$
\Psi(\lambda)=i \lambda\left(e^{-a T} \ln S_{0}+\frac{m}{a}\left(1-e^{-a T}\right)\right)+\int_{0}^{T} \psi\left(\lambda e^{-a(T-s)}-i \theta(s)\right) d s-\int_{0}^{T} \psi(-i \theta(s)) d s .
$$

Proof. The result follows from Sato [12, Lemma 17.1, p.106] or by a direct calculation using the Lévy-Kintchine formula and the independent increment property for the Lévy process $L_{t}$.

Knowing the characteristic function $\psi$ of the Lévy process paves the way for a Fourier approach for pricing options on the spot. We refer to Carr and Madan [7] for a complete description of the fast Fourier transform technique for calculating option prices when the characteristic function of the log-price is known. We end this Section with an example where the characteristic function is calculated when $L_{t}$ is a NIG Lévy process.

4.3. Example: The NIG model. First, let us investigate Condition (L) when the Lévy process $L_{t}$ is of NIG-type.

Lemma 4.5. If $L_{t}$ is a Lévy process where $L_{1}$ is $N I G$ distributed with parameters $(\mu, \alpha, \beta, \delta)$, then Condition $(\mathbf{L})$ is fullfilled for every $k<\alpha-\beta$.

Proof. Inserting the Lévy measure in (2.6) of a NIG Lévy process, we must prove that

$$
\int_{1}^{\infty} \mathrm{e}^{k z} \ell(d z)=\frac{\alpha \delta}{\pi} \int_{\alpha}^{\infty} \mathrm{e}^{\frac{k+\beta}{\alpha} x} \frac{K_{1}(x)}{x} d x
$$

is finite. Appealing to formula 9.6.23 in [1] we can rewrite the integral as

$$
\int_{1}^{\infty} \mathrm{e}^{k z} \ell(d z)=\frac{\alpha \delta}{2 \sqrt{\pi} \Gamma(3)} \int_{1}^{\infty} \sqrt{t^{2}-1} \int_{\alpha}^{\infty} \mathrm{e}^{-\left(t-\frac{k+\beta}{\alpha}\right) x} d x d t
$$

which is finite as long as $k<\alpha-\beta$. Hence the Lemma follows.

From (2.7) we find the moment generating function $\phi(\lambda)=\psi(-i \lambda)$ of a NIG distributed random variable with parameters $(\mu, \alpha, \beta, \delta)$ to be

$$
\phi(\lambda)=\mu \lambda+\delta\left(\sqrt{\alpha^{2}-\beta^{2}}-\sqrt{\alpha^{2}-(\lambda+\beta)^{2}}\right), \quad|\lambda+\beta| \leq \alpha .
$$

The forward price with delivery at time $T$ becomes

$$
\begin{aligned}
F^{\theta}(t, T)=\Lambda(T) & \exp \left(\frac{\mu}{a}\left(1-\mathrm{e}^{-a(T-t)}\right)\right)\left(\frac{S_{t}}{\Lambda(t)}\right)^{\exp (-a(T-t))} \\
& \times \exp \left(\delta \int_{t}^{T}\left\{\sqrt{\alpha^{2}-(\theta(s)+\beta)^{2}}-\sqrt{\alpha^{2}-\left(\theta(s)+\mathrm{e}^{-a(T-s)}+\beta\right)^{2}}\right\} d s\right),
\end{aligned}
$$

for every $\theta$ such that $\sup _{s \in[0, T]}\left|\theta(s)+\mathrm{e}^{-a(T-s)}+\beta\right|<\alpha$.

If we are interested in calculating option prices for a given market price of risk $\theta$ we easily derive the characteristic function of $X_{T}$ with respect to the probability $\mathcal{Q}^{\theta}$ from (2.7) and 
Cor. 4.4 as

$$
\Psi(\lambda)=\mathrm{i} \lambda\left(\mathrm{e}^{-a T} \ln S_{0}+\frac{m}{a}\left(1-\mathrm{e}^{-a T}\right)\right)+\mathrm{i} \lambda \frac{\mu}{a}\left(1-\mathrm{e}^{-a T}\right)+\int_{0}^{T} \Phi(s) d s,
$$

with

$$
\Phi(s)=\delta\left(\sqrt{\alpha^{2}-(\beta+\theta(s))^{2}}-\sqrt{\alpha^{2}-\left(\beta+\left(\theta(s)+\mathrm{i} \lambda \mathrm{e}^{-a(T-s)}\right)\right)^{2}}\right),
$$

for $\theta$ such that $\sup _{s \in[0, T]}|\beta+\theta(s)|<\alpha$.

\section{Conclusions}

We have suggested an exponential non-Gaussian Ornstein-Uhlenbeck model for the spot price of energies. This model is simple to fit to price data compared with alternative models stated as solutions of stochastic differential equations. Motivated by Barndorff-Nielsen [2], we propose to use the normal inverse Gaussian distribution for modelling the residuals in the Ornstein-Uhlenebeck process. The analysis of Brent oil and SAP spot prices shows a clear rejection of the normal hypothesis, while the normal inverse Gaussian distribution fits the data reasonably well and provides a more accurate picture of the actual spot price dynamics.

We have derived explicit forward prices and discussed how to find the market price of risk. As the basic ingredient in an efficient valuation of option prices on spots, we have calculated the characteristic function for the logarithmic spot price.

\section{REFERENCES}

[1] Abramowitz, M. and Stegun, I.A. (1972). Handbook of Mathematical Functions. Dover Publ., New York.

[2] Barndorff-Nielsen, O. E. (1998). Processes of normal inverse Gaussian type. Finance E Stochastics, 2, pp. 41-68.

[3] Barndorff-Nielsen, O. E. and Shephard, N. (2001). Non-Gaussian Ornstein-Uhlenbeck-based models and some of their uses in financial economics. J. R. Stat. Soc. Ser. B Stat. Methodol., 63(2), pp. 167241.

[4] Barndorff-Nielsen, O. E. and Shephard, N. (2003). Financial volatility: Stochastic volatility and Lévy based models. Cambridge Unversity Press, Cambridge.Monograph in preparation.

[5] Benth, F.E., Ekeland, L., Hauge, R., and Nielsen, B.F. (2001). On arbitrage-free pricing of forward contracts in energy market. E-print, No. 14, University of Oslo.

[6] Bjørk, T. (1997). Interest rate theory. Lecture Notes in Math. 1656 Springer, Berlin, pp. 53-122.

[7] Carr, M. and Madan, D.B. (1999). Option valuation using the fast Fourier transform. J. Comp. Finance, 2(4), pp. 61-73.

[8] Clewlow, L. and Strickland, C. (2000). Energy Derivatives. Pricing and Risk Management. Lacima Publications, London.

[9] Doornik, J.A. (1998). Ox: Object Oriented Matrix Programming 2.0. Timberlake, London.

[10] Eydeland, A. and Wolyniec, K. (2003). Energy and Power Risk Management. John Wiley \& Sons, New Jersey.

[11] Pilipović, D. (1998). Energy Risk. Valuing and Managing Energy Derivatives. McGraw-Hill, New York.

[12] Sato, K.-I. (1999). Lévy processes and infinitely divisible distributions. Cambridge University Press. 
[13] Schwartz, E. S. (1997). The stochastic behaviour of commodity prices: Implications for valuation and hedging. J. Finance LII(3) 923-973.

(Fred Espen Benth)

Centre of Mathematics for Applications

Department of Mathematics

UNIVERSITY OF OSLO

P.O. Box 1053, BLINDERN

N-0316 Oslo, Norway

AND

Agder University College

Department of Economics and Business Administration

Serviceboks 422

N-4604 Kristiansand, Norway

E-mail address: fredb@math.uio.no

URL: http://www.math. uio.no/“fredb/

(Jūratè Šaltytè-Benth)

Centre of Mathematics for Applications

Department of Mathematics

UNIVERSITY OF OSLO

P.O. Box 1053, BLINDERN

N-0316 Oslo, Norway

AND

Department of System Research

KLAIPĖDA UNIVERSITY

H. Manto 84

LT-5808 KLAipĖDa, Lithuania

E-mail address: jurate@math.uio.no 